\title{
Review of PMSG
}

\author{
YAN Yujia ${ }^{1, a}$, ZHAO Jincheng ${ }^{1, b}$ \\ ${ }^{1}$ Department of Electric Engineering, Army Engineering University, Shijiazhuang 050003, China \\ a710502810@qq.com, bratyyj@163.com
}

Keywords: Permanent magnet, Synchronous generator, Radial flux, Axial flux.

\begin{abstract}
A review of permanent magnet synchronous generator (PMSG) of different topologies is carried out in the paper. Many configurations are chosen for the comparison, consisting of both radial-flux and axial-flux machines. For a given application, the results provide an indication of the best suitable generator.
\end{abstract}

\section{Introduction}

As the permanent magnet synchronous generator (PMSG) has been suggested for a long time, it is developing very fast in recent years because of the development of the rare earth permanent magnetic materials. According to different classification, there are many different PMSG configurations.

\section{Generator topologies investigated}

All the compared configurations are three phase permanent magnet synchronous generators.

\subsection{Radial flux generator}

- Inner rotor radial flux generator

It is a typical radial flux generator with the PM poles rotating inside the stationary armature windings, as shown in Fig. 1. The stator is made up of electrical grade steel laminations with distributer windings. The rotor is cylindrical in shape with a shaft on which the bearings are mounted. There are two magnets providing the magneto motive force (MMF) required in a pair of poles, which can effectively resist the demagnetization caused by the armature reaction in a sudden short circuit. The air gap flux density is closely related to the magnet remanence and the magnet working point. It is difficult to get high air gap flux densities with low remanence magnets in this configuration.

The radial flux magnet generator is the most common type of PM generator. These machines have a higher torque capability than common induction generator as well as electrically excited synchronous generator. The efficiency is higher than induction generator because it does not have rotor windings, also suggests that it has higher power and torque density. These generators can be easily designed with a high pole number that is required for low speed operations. However, this type of generator has long end windings when the aspect ratio $\mathrm{D} / \mathrm{L}$ is small which could lead to higher copper loss. Also the flux density is reduces due to large gap.

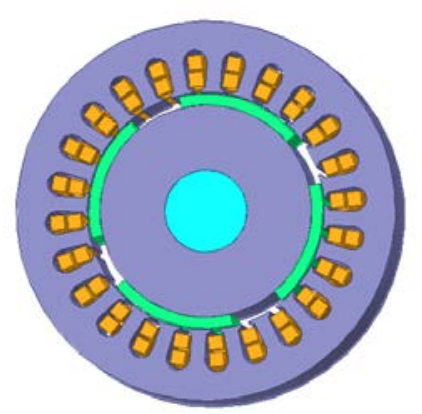

Fig. 1 Inner rotor radial flux generator 
Outer rotor radial flux generator

The wound stator in the outer- rotor configuration is stationary, located at the center of the machine, while the magnets are mounted evenly along the inner circumference of the rotating drum supported by front and rear bearings, as shown in Fig. 2. The magnetic circuits are the same as those in the conventional inner-rotor radial-flux generator. The blades of the wind turbine can be conveniently bolted to the front face of the drum to realize the direct coupling between the wind turbine and the PM generator. Because of the enlarged periphery of the outer-rotor drum, the multi pole structure can be easily accommodated, and therefore the total length of the magnetic path is reduced. As the rotor is directly exposed to the wind, the cooling condition is improved for the magnets so that the resistance to high temperature demagnetization is enhanced.

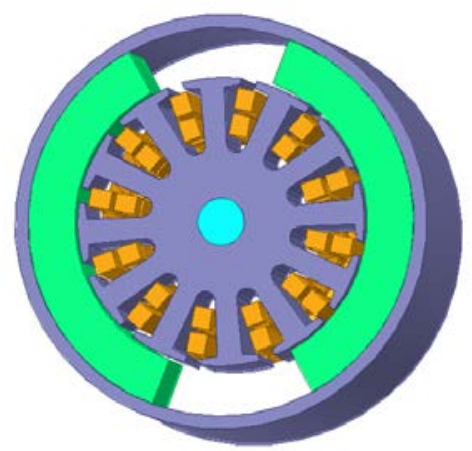

Fig. 2 Outer rotor radial flux generator

\subsection{Axial flux generator}

In an axial flux generator, the rotor rotates relative to the stator with the flux crossing the air gap in the axial direction. These generators can have slot-less windings which allows larger air-gap length. And the AFPG usually requires larger magnet than PFPM leading to higher magnet costs.

There are four common types of AFPG configurations:

- Single rotor and stator

This configuration is simple, as there is only one stator and one rotor as shown in Fig. 3. However, a large attractive force exists between the stator and the rotor. To prevent the rotor from moving in the axial direction, a special thrust bearing must be used, which will make the construction more complicated. By adding an additional stator to the construction, an effective way is introduced to balance the attractive force. On the opposite side of the rotor, PM poles are needed to produce the magnetic field necessary to induce the balance force. The stator is laminated, as the magnetic field oscillates, creating hysteresis and eddy-current losses. When the flux path is carefully designed, the force produced between the rotor and the additional stator can balance the force between the rotor and the slotted machine stator.

Another configuration is similar to the single-sided axial-flux machine with stator balance, except that an additional rotor with mounted magnet is added to the construction instead of a stator balance. The stator yoke length should be extended to provide a path for the magnetic field through which the balance force will be induced. An iron flux path is needed on the additional rotor back of yoke. Thus, this construction uses more materials than the stator balance construction. 


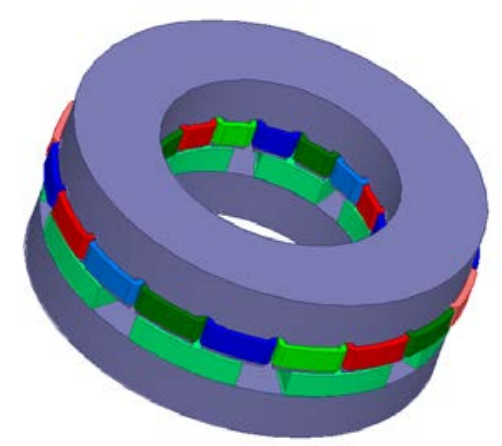

Fig. 3 Single rotor and stator

Double-stator

The shape of the stator as well as the rotor resembles a pancake, and these machines are commonly referred to as pancake machines. The machine consists of two external stators and one inner rotor as shown in Fig. 4. The PMs are axially magnetized and are surface mounted or inset into a cut window on the rotor disc. In all axial flux machines, the rotor rotates relative to the stator with the flux crossing the air gap in the axial direction. The stator iron core is laminated in the radial direction and resembles concentric rings that have a constant slot width and tapered teeth.

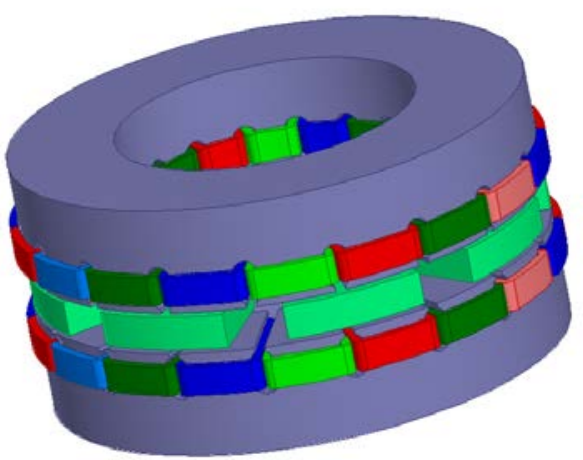

Fig. 4 Double-stator

Double-rotor

This configuration is similar to that of the double-stator slotted axial-flux machine, except that there is one stator and two rotors as shown in Fig. 5. The stator is located in the middle of the two rotors and slotted on both sides. An iron flux path is needed on the rotor back of yoke, but the stator back yoke can be eliminated and saved.

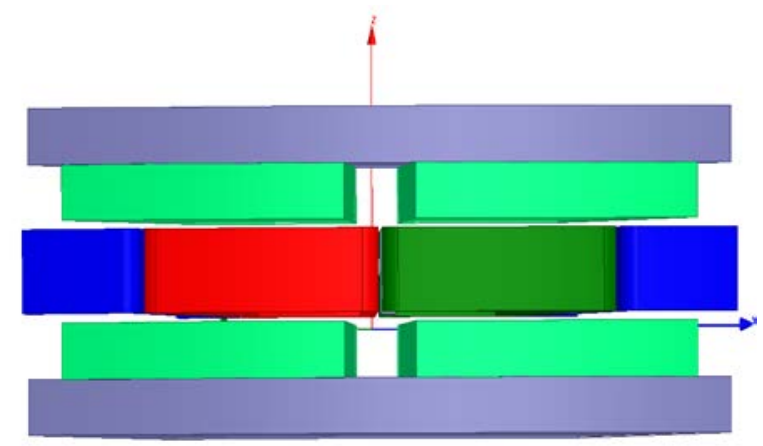

Fig. 5 Double-rotor

Toroidal winding

This kind of prototype generator has a simple construction and is often referred to as a Torus machine. It is a spotless double-sided axial flux PM disc-typed machine. The two rotor discs are made of mild steel and have surface-mounted PMs to produce an axially directed magnetic field in the machine air gaps. The machine stator comprises a spotless toroidally wound strip-iron core that 
carries a three-phase winding in a toroidal fashion by means of concentrated coils. The coils have a rectangular shape according to the core cross section. The axially directed end-winding lengths are relatively short, yielding low resistance and reduced power loss. The active conductor lengths are the two radial portions facing the magnets, the polarities of which are arranged to induce additive electromotive forces (EMFs) around a stator coil.

\section{Comparison in different flux directions}

A comparison was done between the axial flux permanent magnet generator (AFPG) and the radial flux permanent magnet generator (RFPG) which showed that AFPG have greater power density. The result also showed that the double-rotor AFPG has the highest torque density and the singe rotor-stator configuration has the lowest. On the other hand, the double stator AFPG design results in a very compact structure because of elimination of rotor yoke, axial length can be made very short. In summary, the advantages AFPG over RFPG are:

Higher power density, hence requires less core material.

They have planer and easily adjustable air gap.

The noise and vibration are less

Low cogging torque in the slot-less structure

The major disadvantages of AFPG includes, structural instability with large diameter discs, high magnet cost in slot-less design and requirement of large outer diameter makes it difficult to be used in the large scale.

\section{Conclusions}

Different configurations of PMSG have different characteristics, and fit in different places. For example, in order to have the highest power density, double stator machine is the best choice. At the same time, 3D simulation and radiation design are hard problems for further study.

\section{References}

[1] Daniel Fodorean,Lorand Szabo. Study of Permanent Magnet Synchronous Machine Topologies for Electric Scooter Application[J]. Advanced Engineering Forum,2013,2562(8):.

[2] Tao Wu,Ning Jun Fan,Yan Xuan Wu. Analysis of Magnetic Field for Micro Axial-Flux Electromagnetic Generator[J]. Key Engineering Materials,2013,2477(562):

[3] Mei Zhang,Hai Qin Xue,Shui Liang Zhou. A Review on Low Voltage Ride-Through Solutions for PMSG Wind Turbine[J]. Advanced Materials Research,2015,3701(1070):.

[4] Tiara R.S. de Freitas,Paulo J.M. Menegáz,Domingos S.L. Simonetti. Rectifier topologies for permanent magnet synchronous generator on wind energy conversion systems: A review[J]. Renewable and Sustainable Energy Reviews,2016,54:.

[5] T.P.M. Bazzo,J.F. Kolzer,R. Carlson,F. Wurtz,L. Gerbaud. Multidisciplinary design optimization of direct-drive PMSG considering the site wind profile[J]. Electric Power Systems Research,2016,:. [6] Mohsen Rahimi. Modeling, control and stability analysis of grid connected PMSG based wind turbine assisted with diode rectifier and boost converter[J]. International Journal of Electrical Power and Energy Systems,2017,93:.

[7] Zhiwei Zhang,Libing Zhou. Design And Rotor Geometry Analysis Of Permanent Magnet Assisted Synchronous Reluctance Machines Using Ferrite Magnet[J]. Journal of Electrical Engineering,2015,66(6):. 